Recepción: 05 / 07 / 2018

Aceptación: $11 / 09$ / 2018

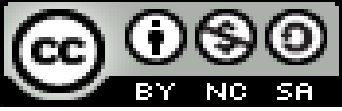

Ciencias de la educación

Publicación: 01 / 11 / 2018

Artículo de investigación

\title{
EI freno inhibitorio en la autorregulación infantil: Análisis de experiencia
}

The inhibitory brake in infantile self-regulation: Analysis of experience

O freio inibitório na auto-regulação infantil: análise da experiência

\author{
Elena Rosero-Morales ${ }^{\mathrm{I}}$ \\ elenadroserom@uta.edu.ec \\ Myrian Pérez-Constante II \\ mb.perez@uta.edu.ec \\ Nereida Camino-Villamarín III \\ ndr.camino@uta.edu.ec
}

\section{Correspondencia: elenadroserom@uta.edu.ec}

\footnotetext{
I Magister en Psicología Educativa, Psicóloga Educativa y Orientadora Vocacional, Docente de la Universidad Técnica de Ambato, Ambato, Ecuador.
${ }^{\text {II }}$ Magister en Pedagogía en Ciencias de la Salud, Licenciada en Estimulación Temprana, Docente de la Universidad Técnica de Ambato, Ambato, Ecuador.

III Magister en Auditoria Gubernamental, Ingeniera en Contabilidad y Auditoría CPA, Docente de la Universidad Técnica de Ambato, Ambato, Ecuador.
} 


\section{Resumen}

La presente investigación tiene como objetivo conocer el freno inhibitorio y su influencia en la autorregulación en niños y niñas de 4 a 5 años dentro del sistema educativo, los instrumentos aplicados fueron una ficha de observación dirigido a 30 niños y niñas con una Ficha Técnica (adaptación al Test de Goldstein) que facilitó la obtención de información de la autorregulación que incluye el análisis de las habilidades sociales, personales e interpersonales, aplicado a 8 docentes. Se ejecutó un estudio de tipo cuantitativo, las modalidades fueron bibliográfica y documental, los niveles de tipo exploratorio, descriptivo, explicativo, utilizados para demostrar que la investigación es especifica; además se generaron datos numéricos con el fin de verificar la hipótesis establecida del freno inhibitorio y su influencia en la autorregulación en niños, concluyendo que las docentes no implementan actividades en favor del freno inhibitorio y la autorregulación, lo que perjudica en la adaptación y aprendizaje.

Palabras clave: autorregulación; conductas; freno inhibitorio; habilidades; adaptación.

\section{Abstract}

The present investigation has as objective to know the inhibitory brake and its influence in the self-regulation in boys and girls of 4 to 5 years within the educative system, the applied instruments were an observation card directed to 30 boys and girls with a Technical File (adaptation to the Goldstein Test) that facilitated the obtaining of self-regulation information that includes the analysis of social, personal and interpersonal skills, applied to 8 teachers. A quantitative study was carried out, the modalities were bibliographic and documentary, the levels of exploratory, descriptive, explanatory type, used to demonstrate that the research is specific; In addition, numerical data were generated in order to verify the established hypothesis of inhibitory inhibition and its influence on self-regulation in children, concluding that teachers do not implement activities in favor of inhibitory inhibition and self-regulation, which impairs adaptation and learning.

Key words: self-regulation; behaviors; inhibitory brake; skills; adaptation.

\section{Resumo}

A presente investigação tem como objetivo conhecer o freio inibitório e sua influência na autorregulação em meninos e meninas de 4 a 5 anos dentro do sistema educativo, os instrumentos 
aplicados foram um cartão de observação direcionado a 30 meninos e meninas com um Arquivo Técnico (adaptação para o Teste Goldstein) que facilitou a obtenção de informações de autorregulação que incluem a análise de habilidades sociais, pessoais e interpessoais, aplicadas a 8 professores. Foi realizado um estudo quantitativo, cujas modalidades foram bibliográficas e documentais, os níveis de tipo exploratório, descritivo, explicativo, utilizados para demonstrar que a pesquisa é específica; além dados numéricos foram gerados, a fim de verificar o freio inibitório hipótese estabelecida e sua influência na auto-regulação em crianças, concluindo que os professores não implementar atividades para o freio inibitório e auto-regulação, o que prejudica a adaptação e aprendizagem.

Palavras chave: auto-regulação; comportamentos; freio inibitório; habilidades; adaptação.

\section{Introducción}

El análisis del freno inhibitorio y la autorregulación es un tema de interés en el estudio de investigadores, docentes, psicólogos, por su relación con el aprendizaje en el entorno educativo definido en un proceso activo que contribuye en la regulación, el control de emociones, motivación, comportamiento y conducta. El presente estudio surge como necesidad para sintetizar una base teórica para respaldar la importancia del freno inhibitorio en la autorregulación en el sistema educativo y de esta manera contribuir en el control de emociones conductas o comportamientos inadecuados que desde la perspectiva psicopedagógica y educativa genera afectaciones en la autorregulación, el rendimiento, creatividad; incluye la inteligencia espacial, psicomotricidad, comportamiento infantil junto con las habilidades socioemocionales que favorecen en la profundización del conocimiento relacionado con el freno inhibitorio.

\section{La Educación, freno inhibitorio y autorregulación.}

En el Ecuador a través de diferentes programas difundidos por el Estado se fomenta el desarrollo de prácticas y experiencias que desde un enfoque integral contribuyen en el progreso del pensamiento, las habilidades intelectuales, beneficiando en la autonomía, el ámbito psicomotriz, el freno inhibitorio, las conductas y comportamientos con el fin de fortalecer la convivencia en un ambiente de armonía y respeto. Para Jaume, Ribot, \& otros (2018) manifiestan que la educación aporta en el desarrollo, aprendizaje, adquisición de experiencias, conocimientos, seguridad, 
autonomía, los valores comunicativos, funcionales, semánticos, estimula el control y autorrealización; es así como la autorregulación se caracteriza por ser un proceso que beneficia en el desarrollo integral, mantiene una visión holística que apoya en el crecimiento humano, al tener como propósito lograr la autonomía e independencia en la solución de problemas, la toma de decisiones; de esta manera se deduce que la educación es un proceso de adquisición de conocimientos, habilidades y hábitos entre un grupo de personas, a través de diferentes actividades recreativas que impulsa la expresión de emociones y pensamientos.

El sistema educativo incluye en la labor pedagógica actividades que el docente debe cumplir para mejorar los procesos de enseñanza-aprendizaje, al tener como meta el progreso infantil, de competencias y potencialidades en un entorno donde el estudiante es el protagonista de su conocimiento. Según Taboada \& Pinto (2018), sustenta que los niños pueden presentar problemas en funciones de atención y cognitivas como, resolución de problemas, orientación, alertas, atención sostenida, inhibición de respuesta, deduciéndose que el desarrollo infantil incluye interacciones que benefician en el crecimiento, madurez y aprendizaje, generando cambios en las habilidades intelectuales y emocionales, que promueven el aprendizaje en Educación Inicial valorada como parte del proceso formativo, al contribuir en el crecimiento de los niños y niñas.

En educación infantil, la aplicación de metodologías innovadoras conlleva al alcance de aprendizajes cooperativos, que mediante la orientación y guía del docente son fortalecidos mediante la aplicación de gráficos, audios, actividades de aspecto lúdico que incluyen la dinámica y creatividad, además la realización de juegos motrices estimula el freno inhibitorio y el autocontrol de la misma manera es importante en el desarrollo intelectual. Para Ríos, Álvarez, Sierra, \& Zuleta (2017) menciona que son más frecuentes los casos de inatención o impulsividad en los niños y niñas percibidos en sus hogares con conductas atípicas, síntomas de hiperactividad, generando afectaciones en los procesos conductuales o comportamentales al influenciar en las habilidades físicas y psicológicas; desde la perspectiva de educación inicial el niño adquiere bases para la autonomía, el autocontrol y la autoestima.

La investigación perteneciente al freno inhibitorio se efectúo como resultados de diversas perspectivas de respuesta al esclarecer ¿Cómo los estudiantes alcanzan la autorregulación?, el proceso indagatorio cumple con los siguientes objetivos: conocer el nivel del freno inhibitorio, 
identificar la autorregulación; determinar la relación del freno inhibitorio en la autorregulación que el infante al lograr el control de su cuerpo, realiza movimientos, alcanza el dominio muscular logrando movimientos coordinados; disminuyendo dificultades en el aprendizaje. El trabajo se deduce que la mayoría de infantes tiene dificultades en el control de movimientos de brazos, piernas y torso, la mayoría pasan sentados en el aula, reciben un instrucción tradicional, es intranquilo, no reconoce su lateralidad, el eje de su cuerpo, la organización de diferencias espaciales/derecha izquierda; mantiene un escaso autocontrol en el manejo de emociones, se muestra impulsivo, emite respuestas inmediatas hacia el llamado de atención, presenta conflictos en el proceso de socialización, se distrae con facilidad creando una rutina de trabajo que aumenta el desinterés en el aprendizaje, es descuidado, se adapta con dificultad al ambiente educativo exterioriza problemas en pensar antes de actuar, actitudes inapropiadas, se comporta de manera agresiva; es desobediente, a veces asimila reglas de disciplina u orientaciones para efectuar tareas, actividades en el salón de clase o trabajar en equipo.

\section{Importancia del Problema}

El problema es importante porque desde la perspectiva de disciplina y su relación con el freno inhibitorio y la autorrealización para Cladellas, Clariana, \& Badía (2015) afirman que los comportamientos característicos de la indisciplina se correlacionan negativa y significativamente con el rendimiento académico; bajo la perspectiva expuesta las docentes aporten en la formación de infantes utilizando metodologías que apoyen en el lenguaje, el freno inhibitorio, el autocontrol; de esta manera se esclarece que en el aprendizaje los niños y niñas regulan el freno inhibitorio amplían el conocimiento y conciencia de sus habilidades y limitaciones, encaminándose hacia la satisfacción personal. (Jaqueira, Lavega, Lagardera, Araujo, \& Rodrigues, 2014), la educación está asociada a aquellas condiciones que garanticen la igualdad de género y el equilibrio emocional de las personas; la activación de actividades de educación emocional y de bienestar social aporta en la intensidad de emociones positivas, negativas y ambiguas, de esta forma el freno inhibitorio, la autorregulación de emociones, el comportamiento, el diálogo, la expresión a través del lenguaje tanto oral como escrito, intervienen en los principio de equidad e igualdad cooperando en la confianza, regulación, coordinación; elementos que favorecen en la educación. 
Según Morales, Romero, Rosas, López, \& Nieto (2015), los niños al ser nombrados por maestros y padres como conductualmente inhibidos (controlados) o desinhibidos (impulsivos), exteriorizan distintos niveles de culpa por incumplir normas, desde esta visión la rebeldía tiene efectos de interacción entre el negativismo o desafío infantil, el escaso freno inhibitorio, la insuficiente autorregulación y el inadecuado comportamiento que al no ser controlados dan origen a la obediencia infantil, trastorno negativo, conducta desinhibida y conductas carentes de autocontrol. En este sentido Morales, Félix, \& Rosas (2015) señalan que las instrucciones claras, establecimiento de reglas, solución de problemas e interacción social positiva disminuyen la conducta negativa, desafiante y agresión infantil; es decir, los problemas de conducta infantil se relacionan con los escenarios educativo y familiar, en niños entre los 4 y 7 años de edad sobresale los arrebatos, oposición, desobediencia, irritabilidad, enojo y resentimiento (escaso control del freno inhibitorio) hacia la autoridad, lo que provoca deterioro en la actividad social, escolar o familiar, perjudicando en la autorrealización.

Durante la educación pre escolar los infantes desarrollan la capacidad de sentir, la agudeza de las emociones como fuente de conexión, siendo así que la inteligencia social percibe la capacidad de controlar los sentimientos propios y de otras personas, motivándose para el manejo del freno inhibitorio, entendida como capacidad para alcanzar la autorregulación al reconocer los sentimientos, adecuar su expresión y contener la propia impulsividad, para Jiménez (2018) afirma que el conocimiento de las propias emociones, es entendida como capacidad de reconocer los sentimientos cuando aparecen implica una introspección o introversión psicológica eficaz para comprenderse a uno mismo, por las razones mencionadas los maestros al aplicar metodologías que aporten en el desarrollo no solamente del pensamiento también del comportamiento y autoestima fomentan la seguridad en la resolución de conflictos y fortalecimiento de relaciones interpersonales; en la misma línea García, Naissir, Contreras, \& Moreno (2015) agrega que todos los problemas emocionales afectan desfavorablemente el desarrollo integral del niño siendo la educación uno de los sectores desfavorecidos; un problema emocional exhibe un escaso control inhibitorio, factor que afecta en la capacidad de aprender, en las habilidades intelectuales o sensoriales, pudiendo ser una incapacidad de mantener el autocontrol, la autorregulación, las relaciones interpersonales; por comportamientos inapropiados o depresión asociándose con conflictos educativos. 
En el entorno educativo uno de los problemas con mayores consecuencias es el inapropiado rendimiento académico que perjudica la calidad de educación agudizándose en un problema donde los estudiantes carecen de habilidades para controlar sus emociones, ser creativos, resolver problemas, autorregular sus sentimientos; sin embargo, al recibir los estudiantes afectividad familiar, recursos escolares, refuerzo en las prácticas de enseñanza mejoran el desempeño académico, resultando interesante la interacción, entre docentes y educandos, razones que interfirieren en el aprendizaje, el contexto escolar, el desarrollo cognitivo y prevención en el ámbito emocional, por ello según Avanzini (2016) La falta de éxito merece ser estudiada por su creciente importancia social, en una sociedad con un índice de escolarización poco elevado, siendo que el fracaso repercute en la personalidad, las dificultades emocionales y sociales en infantes se originan por la falta de capacidades emocionales y sociales, internalizándose en problemas académicos, el inapropiado rendimiento escolar que incrementan aprietos de aprendizaje, con prevalencia en la conducta, atención y depresión.

En la modelación del comportamiento los padres o cuidadores que aplican el castigo físico, trasmiten a los infantes modelos de agresividad; en contraparte los premios por el buen comportamiento e ignorar los malos es una mejor forma de educar, por ello según Urbieta \& Esteinou (2017) la disciplina positiva ayuda a los niños o niñas a entender por qué ciertos comportamientos son aceptables y otros no; la conducta negativa se enfoca en el cumplimiento de órdenes para evitar castigos que posteriormente generan dificultades en el control de su actuación, por esta razón los maestros al aplicar metodologías para el freno inhibitorio desde el enfoque psicomotriz, el movimiento, la lateralidad, coordinación de las partes del cuerpo, contribuyen en la regulación del proceso de aprendizaje, por otra parte Araque (2017) menciona que la educación emocional es un pilar fundamental en educación inicial para que los niños/as puedan experimentar un desarrollo emocional y social que les permita ser más autónomos, dentro de un marco de convivencia armónica; aspecto esencial que beneficia en la comunicación y adaptación al proceso de enseñanza, Torrano, Fuentes, \& Soria (2017) manifiestan que en el aprendizaje, la aplicación de la técnica del modelado es otro procedimiento para la enseñanza de las estrategias de autorregulación, ya que el ser humano puede adquirir numerosas destrezas, actitudes y conductas a través de la imitación; lamentablemente la insuficiente utilización de metodologías que apoyen en el control de movimientos y coordinación limita el desarrollo de 
destrezas, el freno inhibitorio y experiencias mentales. Para Navea (2017), el aprendizaje autorregulado incluye la utilización de estrategias de aprendizaje y motivación que benefician en los procesos de construcción de conocimiento, Muñoz (2017), incorpora la autorregulación en el apego durante las etapas de la niñez, desde la perspectiva de Fonagy, Gergely, Jurist, \& Target (2002), en edades tempranas los niños desarrollan mecanismos que controlan el freno inhibitorio las conductas y actitudes inapropiadas; Kopp (1982) el proceso de control de comportamientos inapropiados incrementa la satisfacción de necesidades individuales, los procesos mentales intencionales, la disciplina, creatividad y motivación; así, en correspondencia a la autorregulación y freno inhibitorio Aldrete, Carrillo, Mansilla, Schnaas, \& Esquivel (2013) incluyen el autocontrol, las funciones ejecutivas, el control inhibitorio, Castro \& Casado (2014) distinguen la autorregulación conductual y autorregulación emocional (procesos extrínsecos e intrínsecos) para modificar reacciones emocionales, su intensidad y alcanzar metas personales; por otra parte, la autorregulación conductual es la inhibición de las reacciones impulsivas del comportamiento.

Tourón, Santiago, \& Díez (2015) afirman que los estudiantes más capaces muestran, en general, inusual profundidad emocional, intensos sentimientos y reacciones muy sensibles, siendo insuficiente control de emociones lo que afecta en la autorrealización, la autosuficiencia, el desarrollo en las áreas cognitivas; además, poseen la capacidad de relacionar ideas retienen información con mayor facilidad, el vocabulario, comprensión de palabras, autorregulación de emociones, pensamiento intuitivo, sentido de la justicia, concentración; actitudes en las cuales la educación e inteligencia emocional se enlazan en el contexto educativo, el desarrollo evolutivo y socioemocional García \& Barrio (2015) la Educación Infantil, es un momento privilegiado para el aprendizaje de habilidades sociales; las normas o pautas de comportamiento definidas contribuyen a la creación de un ambiente favorecedor de convivencia positiva; en relación a lo expuesto, el desarrollo emocional y convivencia trasciende en la interacción, sociabilización, realización de actividades, el autodominio y autocontrol, de esta manera se determina que el acceso de niños y niñas al sistema educativo mediante el Currículo de Educación Inicial busca el desarrollo de las capacidades cognitivas que viabilicen en el proceso de instrucción, teniendo como enfoque la pedagogía crítica que ubica al educando como actor de su aprendizaje. Para Sáiz \& Pérez (2016), afirman que el concepto de autorregulación del aprendizaje tiene cada vez más 
peso en la investigación psicológica y pedagógica ya que se relaciona con la mejora en el rendimiento académico, es así como la instrucción educativa se encamina en al desarrollo de la inteligencia, las habilidades sociales y autorrealización siendo así que los infantes aprenden a aceptarse, desarrollan su identidad, mejoran su comportamiento, la conducta, el conocimiento, buscan el equilibrio de emociones, autorregulan su aprendizaje mediante procesos cognitivos y meta cognitivo Páramo, Martínez, \& Raposo (2015), el niño aprende desde un nivel inicial de conocimiento, esto permite el desarrollo de competencias, por esta razón se refuerza el control de sus impulsos, el reconocimiento de conductas, la capacidad de relacionarse socialmente a través de experiencias afectivas, influyentes en el desarrollo cognitivo, social y emocional. Demostrando que deberán existir más investigaciones que favorezcan la potencialidad del freno inhibitorio y la regulación infantil.

\section{Metodología}

La población y muestra se constituyó por 30 niños y niñas de 4 a 5 años y 8 docentes del Nivel Inicial de una Unidad Educativa en Tungurahua.

Tabla 1. Población

\begin{tabular}{|c|c|c|}
\hline Objeto de estudio & Frecuencia & Porcentaje \\
\hline Niños y niñas & 30 & $79 \%$ \\
\hline Docentes & 8 & $21 \%$ \\
\hline Total & 38 & 100 \\
\hline
\end{tabular}

Fuente: Elena. R, Myrian. P, Nereida, C, Ecuador, 2018

En el trabajo de investigación, no se aplicó el cálculo de la muestra considerando que la población es pequeña, efectuándose el trabajo con una totalidad de 30 niños y niñas junto con 8 docentes. 
Análisis de fiabilidad: Ficha de observación

\section{Escala: TODAS LAS VARIABLES}

Resumen del procesamiento de los casos
\begin{tabular}{|ll|r|r|}
\hline & & \multicolumn{1}{|c|}{$\mathrm{N}$} & \multicolumn{1}{|c|}{$\%$} \\
\hline Casos & Válidos & 30 & 100,0 \\
& Excluidos & \\
& Total & 0 &, 0 \\
& 30 & 100,0 \\
\hline
\end{tabular}

a. Eliminación por lista basada en todas las variables del procedimiento.

Gráfico 1. Análisis de fiabilidad: Escasa de variables

\section{Estadísticos de fiabilidad}

\begin{tabular}{|c|c|c|}
\hline $\begin{array}{c}\text { Alfa de } \\
\text { Cronbach }\end{array}$ & $\begin{array}{c}\text { Alfa de } \\
\text { Cronbach } \\
\text { basada en } \\
\text { los } \\
\text { elementos } \\
\text { tipificados }\end{array}$ & $\begin{array}{l}\mathrm{N} \text { de } \\
\text { elementos }\end{array}$ \\
\hline 993 & 993 & 10 \\
\hline
\end{tabular}

Fuente: Elena. R, Myrian. P, Nereida, C, Ecuador, 2018

La población investigada se encontró conformada por 30 niños y niñas de 4 a 5 años, obteniéndose en el resumen del procesamiento que todos los casos son válidos; es decir no existe exclusión por lista basada en todas las variables del procedimiento.

Ficha técnica (adaptación de Goldstein). Resumen del procesamiento de los casos

Grafico 2. Análisis de fiabilidad: Escala de variables

\section{Escala: TODAS LAS VARIABLES}

\begin{tabular}{|c|c|c|c|}
\hline \multicolumn{4}{|c|}{ Resumen del procesamiento de los casos } \\
\hline & & $\mathrm{N}$ & $\%$ \\
\hline \multirow[t]{3}{*}{ Casos } & Válidos & 8 & 100,0 \\
\hline & Excluidos $^{a}$ & 0 & , 0 \\
\hline & Total & 8 & 100,0 \\
\hline
\end{tabular}

Fuente: Elena. R, Myrian. P, Nereida, C, Ecuador, 2018 
La población se integró por 8 docentes de Educación Inicial de la Unidad Educativa en Tungurahua, obteniéndose en el resumen del procesamiento que todos los casos son válidos; es decir no existe exclusión por lista basada en todas las variables del procedimiento.

Grafico 3. Resumen del procesamiento de los casos

Estadísticos de fiabilidad

\section{Estadísticos de fiabilidad}

\begin{tabular}{|c|c|c|}
\hline $\begin{array}{l}\text { Alfa de } \\
\text { Cronbach }\end{array}$ & $\begin{array}{c}\text { Alfa de } \\
\text { Cronbach } \\
\text { basada en } \\
\text { los } \\
\text { elementos } \\
\text { tipificados }\end{array}$ & $\begin{array}{c}\mathrm{N} \text { de } \\
\text { elementos }\end{array}$ \\
\hline 974 & 980 & 10 \\
\hline
\end{tabular}

Fuente: Elena. R, Myrian. P, Nereida, C, Ecuador, 2018

En los resultados de la encuesta aplicada a maestros de Educación Inicial de una Unidad Educativa en Tungurahua, mediante el coeficiente Alfa de Cronbach, se obtiene 0,98 alcanzando el criterio de excelente tratándose de un instrumento fiable para su aplicación.

La aplicación de la ficha de la observación y la ficha técnica (adaptación de Goldstein) se realizó mediante la fiabilidad del coeficiente Alfa de Cronbach, obteniendo una consistencia interna que varía entre los valores 0 y 1 , determinándose una correlación entre 0 y 1 índice de solidez interna excelente; mientras mayor sea el acercamiento a 1, mejor será la fiabilidad establece los resultados 0,7 aceptable, 0.8 bueno, 9 excelente menor a 6 no es aceptable.

La población investigada se encontró conformada por 8 docentes de 4 a 5 años de una Unidad Educativa en Tungurahua, con un análisis de factibilidad basada en los resultados estadísticos de 10 ítems, el Alfa de Cronbach de ,974; y en elementos tipificados 0,980, por encontrarse cerca de 1, la Ficha técnica (adaptación de Goldstein) es aprobada con un criterio de excelente; es decir es un instrumento fiable. 


\section{Estadística descriptiva}

Para verificar la hipótesis (el freno inhibitorio y su influencia en la autorregulación en niños y niñas de 4 a 5 años, donde se aplicó la estadística descriptiva utilizándose el modelo lógico presentando la hipótesis nula: el freno inhibitorio no influye en la autorregulación en niños y niñas de 4 a 5 años junto con la hipótesis alternativa: El freno inhibitorio influye en la autorregulación en niños y niñas de 4 a 5 años en la Unidad Educativa en Tungurahua; en el modelo estadístico se aplicó un nivel de significación $\alpha=0.05$; mientras la confiabilidad fue el $95 \%$ y un error de $0,5 \%$.

\section{Distribución de probabilidad}

En el estudio se realizó el análisis de consistencia interna con sustento en el muestreo probabilístico aleatorio de 10 ítems que incluyen alternativas: siempre (4); casi siempre (3), a veces (2), nunca (1).

Los datos obtenidos fueron recabados en la institución durante la jornada académica; entre las actividades efectuadas sobresalieron: orientación a docentes en referencia a la utilización del cuestionario estructurado, de igual manera se fortaleció la honestidad en las respuestas, concientización en la participación, colaboración de forma autónoma y anónima para conseguir la fiabilidad en los resultados.

\section{Resultados}

Se inició consolidando el análisis e interpretación de datos que fueron presentados mediante el uso de estadística descriptiva con un enfoque argumentativo, lo que benefició en la asociación entre las variables. Mediante el empleo del Programa SPSS, se efectuó el análisis estadístico y gestión de información, para realizar el proceso analítico, obteniéndose como resultado la relación entre las variables freno inhibitorio y autorregulación.

Como resultados relevantes se obtuvo el $36 \%$ mantiene un limitado control de movimientos, el $33 \%$ permanece inactivo, un $44 \%$ hiperactivo, el $43 \%$ reacciona ante estímulos, $33 \%$ emite respuesta inmediata frente al llamado de atención y el $47 \%$ se distrae con fiabilidad es intranquilo en el aula, es impulsivo. Los niños en el 50\%, presentan dificultades en atención, cooperación, 
participación en diálogos y conversaciones; el 63\% no es solidario, con menor frecuencia intercambia juguetes, es inseguro, no ha desarrollado cualidades y comportamientos, es insensible; un 50\% reacciona de forma inapropiada cuando lo corrigen, presenta un inadecuado control de emociones. Según el análisis estadístico del programa SPSS se obtiene una fiabilidad del 99\% trabajándose con una confiabilidad del 95\% u nivel de significación del 0,05\% todas las interrogantes fueron válidas para el análisis.

Grafico 4. Resumen del procesamiento de los casos

\begin{tabular}{|c|c|c|c|c|c|c|c|}
\hline \multicolumn{8}{|c|}{ Resumen del procesamiento de los casos } \\
\hline & \multirow[b]{3}{*}{ Prequnta7 } & \multicolumn{6}{|c|}{ Casos } \\
\hline & & \multicolumn{2}{|c|}{ Válidos } & \multicolumn{2}{|c|}{ Perdidos } & \multicolumn{2}{|c|}{ Total } \\
\hline & & $\mathrm{N}$ & Porcentaje & $\mathrm{N}$ & Porcentaje & N & Porcentaje \\
\hline Pregunta3 & 1,00 & 3 & $100,0 \%$ & 0 & $0,0 \%$ & 3 & $100,0 \%$ \\
\hline & 2,00 & 4 & $100,0 \%$ & 0 & $0,0 \%$ & 4 & $100,0 \%$ \\
\hline & 3,00 & 9 & $100,0 \%$ & 0 & $0,0 \%$ & 9 & $100,0 \%$ \\
\hline & 4,00 & 14 & $100,0 \%$ & 0 & $0,0 \%$ & 14 & $100,0 \%$ \\
\hline
\end{tabular}

Fuente: Elena. R, Myrian. P, Nereida, C, Ecuador, 2018

Se observa en la tabla de resumen de procesamiento de casos que los 30 son válidos, es decir no existen casos perdidos en la variable independiente freno inhibitorio, ni en la variable dependiente autorrealización, trabajándose con la totalidad equivalente a un porcentaje del 100\%

Gráfico 5. Medidas Simétricas

\begin{tabular}{|c|c|c|c|c|c|}
\hline \multicolumn{6}{|c|}{ Medidas simétricas } \\
\hline & & Valor & $\begin{array}{l}\text { Error típ. } \\
\text { asint. }^{\text {a }}\end{array}$ & T aproximada ${ }^{\mathrm{b}}$ & $\begin{array}{c}\text { Sig. } \\
\text { aproximada }\end{array}$ \\
\hline Nominal por nominal & $\begin{array}{l}\text { Coeficiente de } \\
\text { contingencia }\end{array}$ &, 848 & & &, 000 \\
\hline Intervalo por intervalo & $R$ de Pearson & ,986 &, 012 & 31,047 &, $000^{\mathrm{c}}$ \\
\hline Ordinal por ordinal & Correlación de Spearman & ,997 &, 003 & 72,021 &, $000^{c}$ \\
\hline $\mathrm{N}$ de casos válidos & & 30 & & & \\
\hline
\end{tabular}

Fuente: Elena. R, Myrian. P, Nereida, C, Ecuador, 2018

Se observa en cada uno de los grupos que el tamaño de la muestra es menor a 50 datos por tanto no se puede aplicar Kolmogorov Smirnov. Visualizándose que la significación asintótica en la Relación de Pearson 0,012 es inferior a 0,05 y la Correlación de Spearman es 0.003 inferior a 0,05 . 
Obteniendo como resultado que con una significación aproximada de 0,00 inferior a 1 nivel de significación de 0,05 y el $95 \%$ de confiabilidad se asume la hipótesis alternativa: El freno inhibitorio influye en la autorregulación en niños y niñas de 4 a 5 años de la Unidad Educativa en Tungurahua.

Grafico 7. Resumen de prueba de hipótesis

\section{Resumen de prueba de hipótesis}

\begin{tabular}{|llll|}
\hline \multicolumn{1}{|c}{ Hipótesis nula } & \multicolumn{1}{c}{ Test } & Sig. & Decisión \\
\hline 1 & $\begin{array}{l}\text { Las categorías de Pregunta3 se } \\
\text { producen con probabilidades } \\
\text { guales. }\end{array}$ & $\begin{array}{l}\text { Prueba de chi- } \\
\text { cuadrado de } \\
\text { una muestra }\end{array}$ &, $016 \begin{array}{l}\text { Rechazar la } \\
\text { hipótesis } \\
\text { nula. }\end{array}$ \\
\hline $2 \begin{array}{l}\text { Las categorías de Pregunta7 se } \\
\text { producen con probabilidades } \\
\text { iguales. }\end{array}$ & $\begin{array}{l}\text { Prueba de chi- } \\
\text { cuadrado de } \\
\text { una muestra }\end{array}$ &, $016 \begin{array}{l}\text { Rechazar la } \\
\text { hipótesis } \\
\text { nula. }\end{array}$ \\
\hline
\end{tabular}

Se muestran las significancias asintóticas. El nivel de significancia es ,05.

Fuente: Elena. R, Myrian. P, Nereida, C, Ecuador, 2018

Según Villanueva (2016) mientras mayor sea el acercamiento a 1, mejor será la fiabilidad establece los resultados 0,7 aceptable, 0.8 bueno, 9 excelente menor a 6 no es aceptable.

La población investigada se encontró conformada por 30 niños y niñas de 4 a 5 años de la Unidad Educativa en Tungurahua, con un análisis de factibilidad basada en los resultados estadísticos de 10 ítems, el Alfa de Cronbach de ,993; y en elementos tipificados 0,993, por encontrarse cerca de 1, la ficha de observación es aprobada con un criterio de excelente; es decir es un instrumento fiable.

\section{Estadística descriptiva}

Para verificar la hipótesis (el freno inhibitorio en niños y niñas de 4 a 5 años de la Unidad Educativa en Tungurahua), se aplica la estadística descriptiva y se utilizó el modelo lógico presentándo la hipótesis nula: el freno inhibitorio no influye en la autorregulación en niños y niñas de 4 a 5 años junto con la hipótesis alternativa: El freno inhibitorio si influye en la autorregulación en niños y niñas de 4 a 5 años de la Unidad Educativa en Tungurahua; en el modelo estadístico se aplicó un nivel de significación $\alpha=0.05$; mientras la confiabilidad fue el $95 \%$ y un error de $0,5 \%$. En el resumen de la prueba de hipótesis de Chi cuadrado de una muestra 
se obtiene como significación 0,016 , por ser menor a 0,05 se rechaza la hipótesis nula y se acepta la hipótesis alternativa: El freno inhibitorio si influye en la autorregulación en niños y niñas de 4 a 5 años de la Unidad Educativa en Tungurahua.

A partir del proceso de recolección de información y datos de la investigación realizada se pueden analizar los siguientes resultados obtenidos de la ficha de observación aplicada a de 4 a 5 años de la Unidad Educativa en Tungurahua, obteniendo como resultados relevantes los siguientes:

Grafico 8. El niño/a controla sus movimientos gruesos.

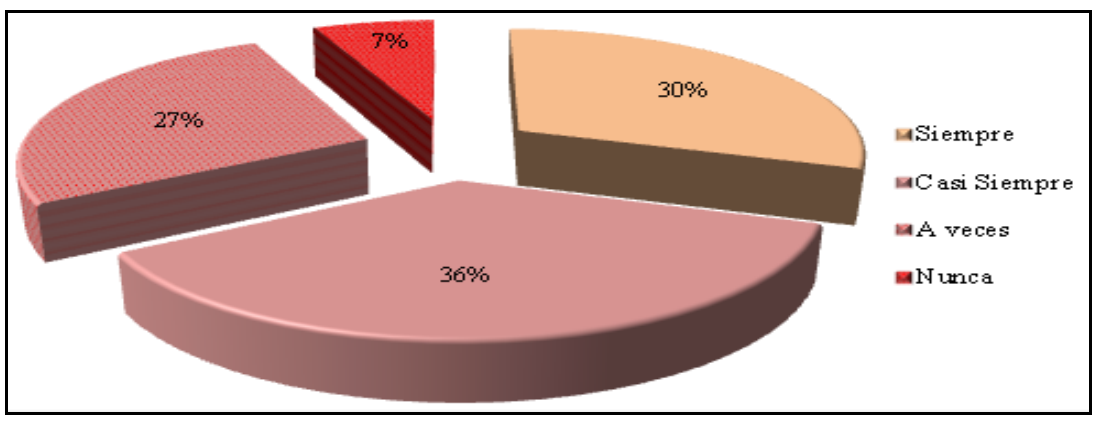

Fuente: Elena. R, Myrian. P, Nereida, C, Ecuador, 2018

Se establece que los niños y niñas presentan dificultades en el control de movimientos de: brazos, piernas y torso, lo que perjudica en la lateralidad, el control de impulsos, la autorregulación, generando comportamientos inadecuados visualizándose sentimientos de antipatía, agresión y desconformidad en el ambiente educativo.

Grafico 9. El niño/a pasa en el aula la mayor parte del tiempo sentado

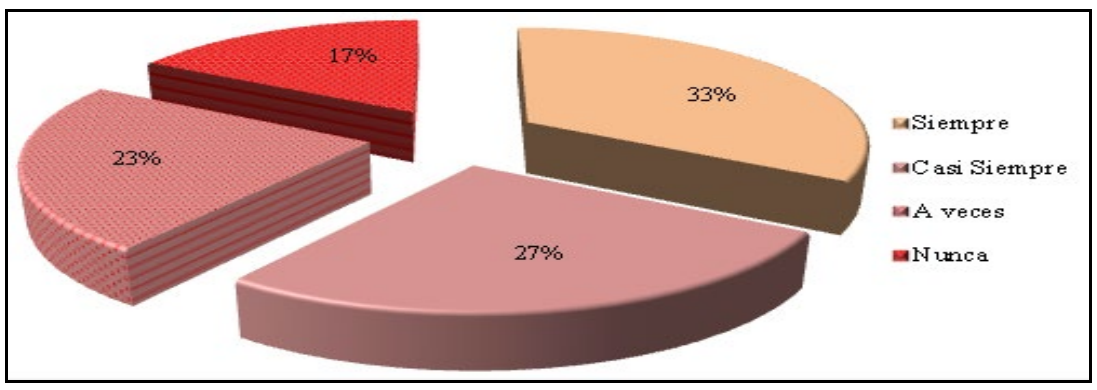

Fuente: Elena. R, Myrian. P, Nereida, C, Ecuador, 2018 
La mayoría de niños y niñas siempre pasan sentados en el aula, reciben un aprendizaje tradicional, sustentado en la repetición de actividades en el aula, lo que perjudica en la participación, el desarrollo del pensamiento crítico, la creatividad e imaginación, contribuyendo en una formación de personas con escasa autoestima, valoración y confianza en sí mismo.

Grafico 10. El niño/a es intranquilo en clase.

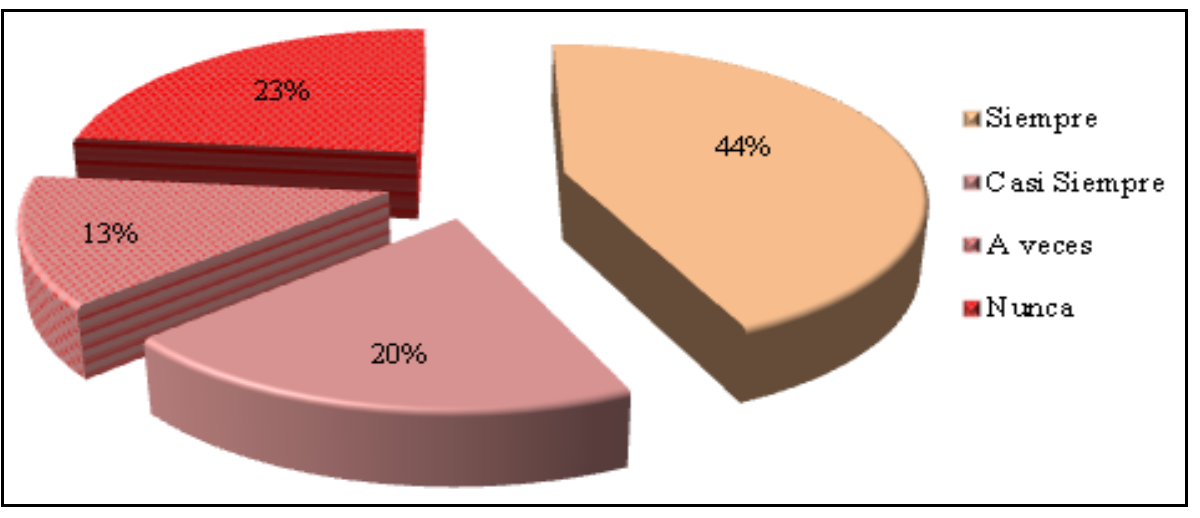

Fuente: Elena. R, Myrian. P, Nereida, C, Ecuador, 2018

La mayoría de infantes demuestra intranquilidad, determinando que la escuela no responde a las necesidades educativas de aprendizaje y crecimiento, no estimula ni activa la atención selectiva de los niños y niñas, como resultado se desplazan y hablan en forma continúa generando conflictos entre ellos; además algunos infantes también se muestran somnolientos, fuera de tema, desinteresados y con poco entusiasmo por participar, sintetizándose que necesitan apoyo para nivelar sus dispositivos básicos de aprendizaje y para ellos se proponen juegos de este tipo que intentarán entrar en el mundo del movimiento controlado.

Grafico 11. El niño/a reconoce su lateralidad.

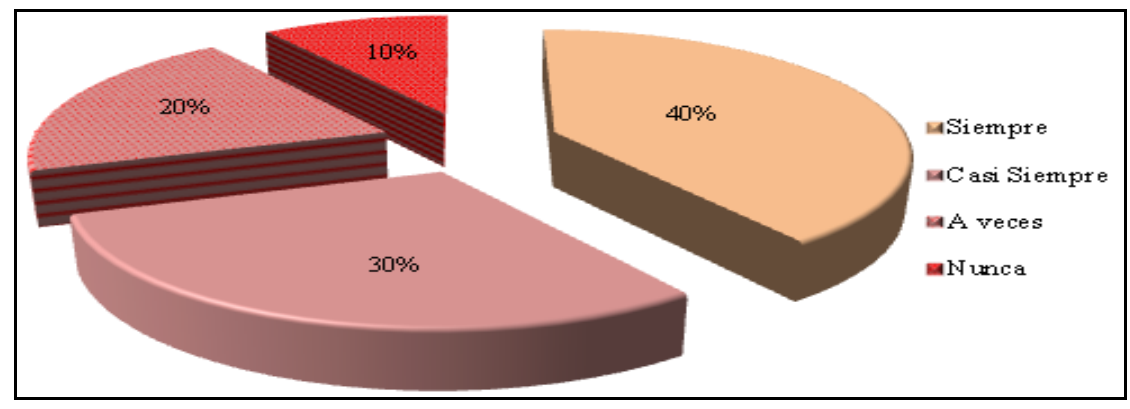

Fuente: Elena. R, Myrian. P, Nereida, C, Ecuador, 2018 
Un porcentaje significativo de niños y niñas reconoce su lateralidad, es decir percibe el eje de su cuerpo y la relación con el entorno, sin embargo, se considera necesario en las etapas de educación infantil se debe estimular la actividad sobre ambas partes del cuerpo con la finalidad de lograr la orientación del cuerpo en el espacio estando en movimiento o en reposo.

Grafico 12. El niño/a reacciona ante un impulso de sus compañeros.

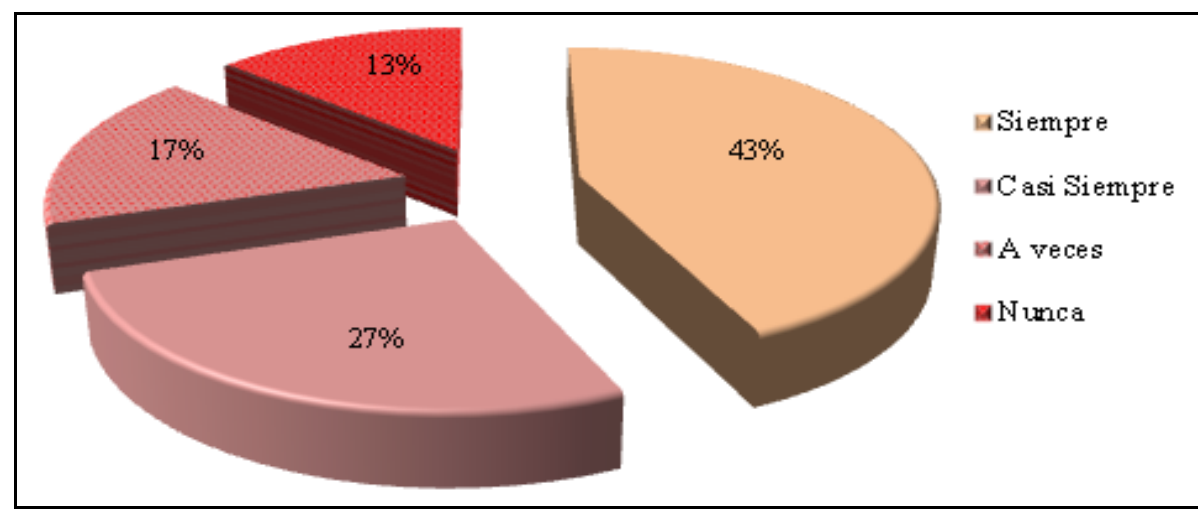

Fuente: Elena. R, Myrian. P, Nereida, C, Ecuador, 2018

Se determina que un porcentaje significativo de infantes siempre reacciona ante el impulso de sus compañeros, necesitan una orientación para expresar, un menor porcentaje casi siempre dominan las emociones y conductas; el otro porcentaje a veces mantiene el autocontrol y confianza en sí mismo; un menor porcentaje nunca reacciona ante impulso, maneja emociones, y relaciones en el aula.

Grafico 12. El niño/a emite respuestas inmediatas hacia el llamado de atención.

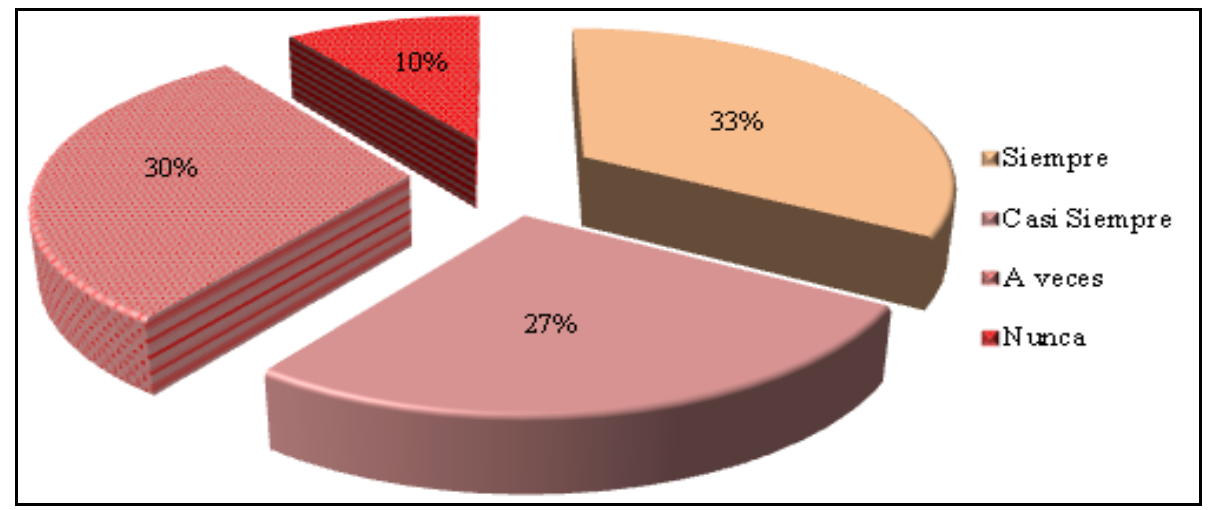

Fuente: Elena. R, Myrian. P, Nereida, C, Ecuador, 2018 
Según los datos estadísticos, los infantes siempre emiten respuestas inmediatas hacia el llamado de atención, al ser parte del desarrollo normal, está estimulando las habilidades lingüísticas; una minoría casi siempre reacciona de manera inmediata, no participa en conversaciones; otros infantes nunca presentan dificultades en el proceso de socialización.

Grafico 13. El niño/a se distrae con facilidad.

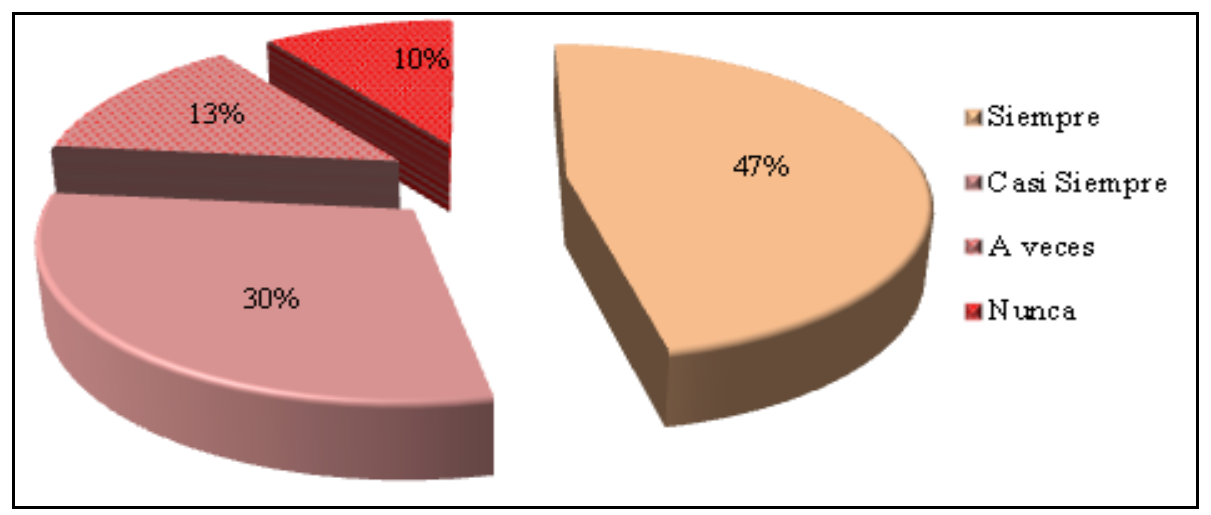

Fuente: Elena. R, Myrian. P, Nereida, C, Ecuador, 2018

Con sustento en el análisis estadístico se observa que los infantes se distraen con facilidad creando una rutina de trabajo que aumenta el desinterés en el aprendizaje; otro porcentaje casi siempre demuestra desatención, lo que perjudica en la ejercitación de la memoria, otros infantes a veces observan detalles es descuidado, presenta dificultades para jugar con tranquilidad, y una minoría nunca se distrae, fortalece su comportamiento en el área educativa.

Grafico 14. El niño/a demuestra tranquilidad en el salón de clase

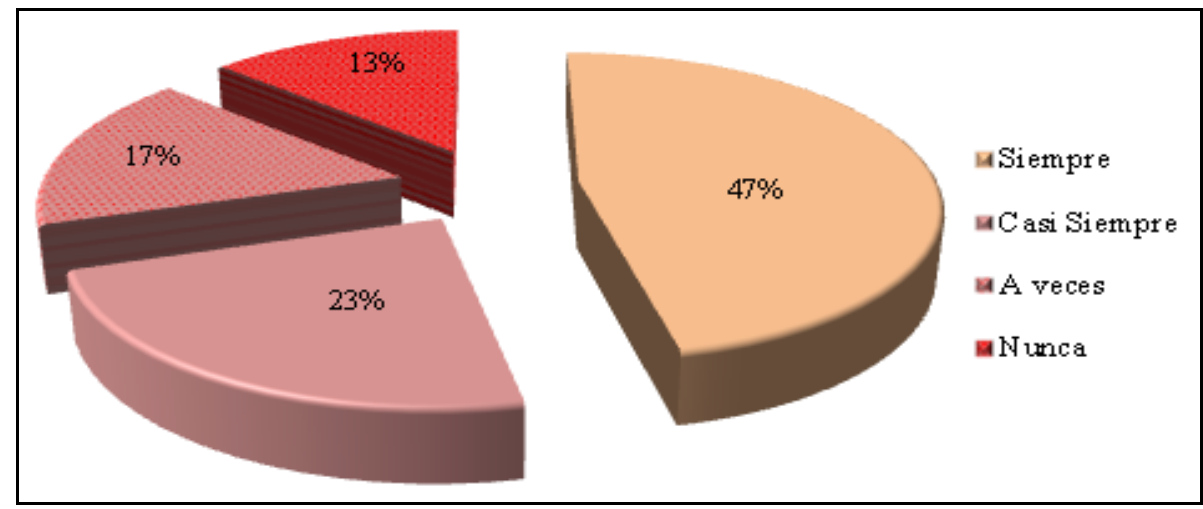

Fuente: Elena. R, Myrian. P, Nereida, C, Ecuador, 2018

En el presente ítem se obtiene que el niño siempre demuestra tranquilidad adoptándose con dificultad al ambiente educativo, otros infantes casi siempre son tranquilos, dóciles y flexibles, un 
menor porcentaje a veces es relajado aprende habilidades para defenderse en el futuro y una minoría nunca demuestra equilibrio y paciencia, lo que afecta en el proceso cognoscitivo.

Grafico 15. El niño/a se muestra impulsivo con sus compañeros.

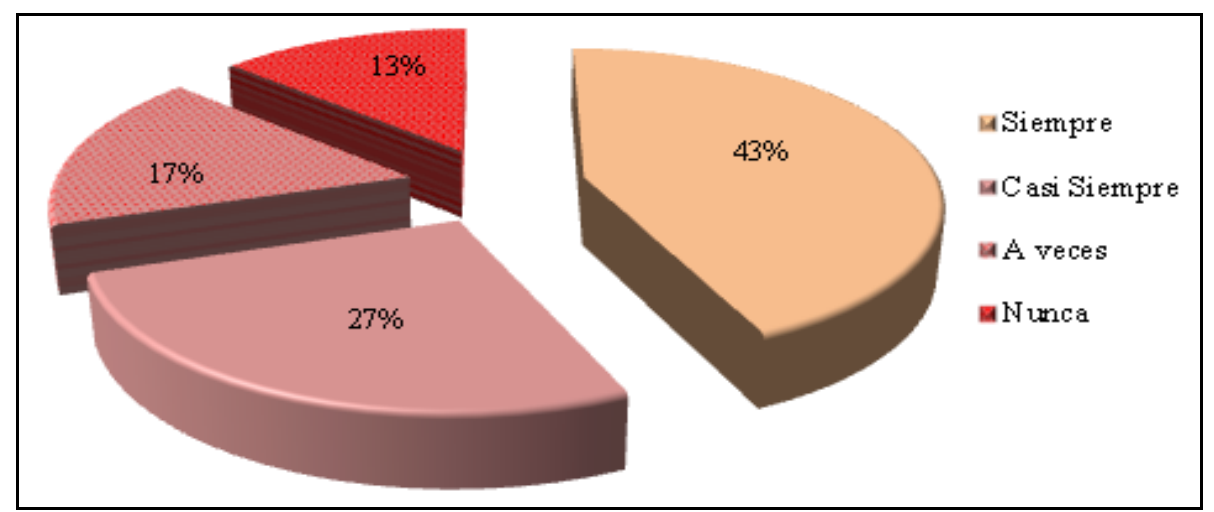

Fuente: Elena. R, Myrian. P, Nereida, C, Ecuador, 2018

En la interrogante se deduce que los niños y niñas exteriorizan conductas impulsivas, no controlan sus emociones antes de actuar; el otro porcentaje casi siempre tiene problemas para pensar antes de actuar, un menor porcentaje a veces realiza cosas inapropiadas para atraer la atención y una minoría nunca se comporta de manera agresiva, ha desarrollado el autocontrol y habilidades sociales.

Grafico 16. El niño sigue instrucciones de su maestra.

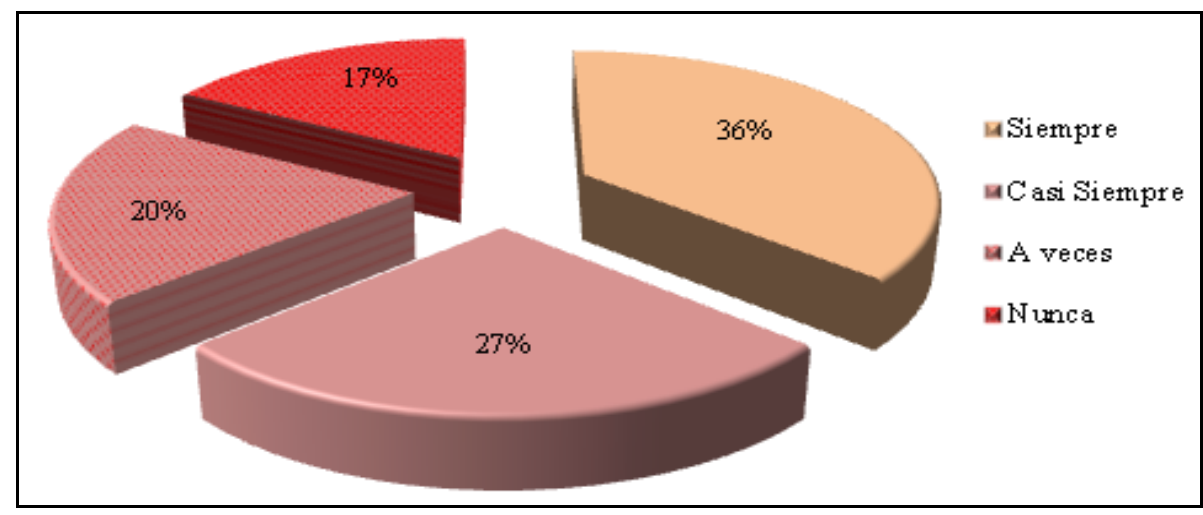

Fuente: Elena. R, Myrian. P, Nereida, C, Ecuador, 2018

En la gráfica presentada se visualiza que los niños y niñas siempre siguen instrucciones, obedece, demuestra una actitud de colaboración; una minoría casi siempre controla y regula su conducta; el otro porcentaje a veces asimila reglas y una minoría nunca cumple sus obligaciones y reglas, lo que desfavorece en la interacción social. 
Un porcentaje significativo presenta dificultades en el control de movimientos de: brazos, piernas y torso; un 33\% pasa sentados en el aula, recibe un aprendizaje tradicional que perjudica en la atención, el comportamiento y rendimiento cognitivo; el $44 \%$ de niños es intranquilo, determinando que la escuela no responde a las necesidades educativas de aprendizaje y crecimiento, no estimula ni activa la atención selectiva de los niños y niñas, como resultado se desplazan y hablan en forma continua generando conflictos entre ellos. El $40 \%$ exterioriza limitaciones en el reconocimiento de su lateralidad, la organización de diferencias espaciales /derecha izquierda lo que afecta en el dominio del esquema corporal y la orientación espacial; en un $43 \%$ los infantes reaccionan ante el impulso de sus compañeros, necesitan una orientación para expresar, dominar las emociones y conductas para alcanzar el autocontrol y confianza en sí mismo.

Mientras en las dimensiones habilidades sociales, conductas y control de impulsos, el 33\% emiten respuestas inmediatas hacia el llamado de atención, al ser parte del desarrollo normal, está estimulando las habilidades lingüísticas; un $47 \%$ de niños y niñas se distraen con facilidad creando una rutina de trabajo que aumenta el desinterés en el aprendizaje; demuestra desatención, lo que perjudica en la ejercitación de la memoria, es descuidado, presenta dificultades para integrarse al grupo de trabajo durante la jornada académica, disminuyendo el proceso cognitivo, intelectual, y razonamiento; además, un 43\% exteriorizan conductas impulsivas, no controlan sus emociones antes de actuar, siempre tiene problemas para pensar antes de actuar, demuestra conductas inapropiadas para atraer la atención, comportándose de manera agresiva, lo que condiciona en el desarrollo del autocontrol y habilidades sociales. De acuerdo a los resultados conseguidos se induce que el freno inhibitorio se caracteriza por controlar comportamientos impulsivos, mediante el manejo de relaciones el conocimiento, la comprensión de sentimientos, sin embargo, el escaso desarrollo del freno inhibitorio afecta en la autorregulación, perjudica en la reflexión para solucionar problemas, tomar conciencia del estado de su conocimiento, motivación, interviniendo en la auto observación, la regulación de impulsos y rendimiento escolar. 


\section{Discusión}

En relación a la atención que presentan los niños y niñas los maestros mencionan que no atiende a las personas cuando hablan, lo que perjudica en el procesamiento de información codificación y estructuración, es decir el niño o niña no controla la impulsividad se distraen por estímulos externos. Para Regni (2014) menciona que estudios sobre la capacidad de atención y su relación con la memoria confirman que es necesario proporcionar a los niños la posibilidad de concentrarse durante largo tiempo en una actividad con materiales adecuados y sin interrupciones, mientras Morales, Arriaga, Peña, \& Mandujano (2017) comenta que los problemas graves de conducta pueden asociarse con una carencia de respuesta, por parte de los niños, al contexto asociado con la ocurrencia del castigo Cano \& Casado (2015), en este sentido el buen maestro debe conocer las situaciones emocionales por las que transitan las familias a lo largo del proceso educativo y saber orientar potenciando buena relaciones entre la familia; en concreto las instrucciones expresadas con claridad y utilizando un lenguaje de acuerdo a la edad del infante, al igual que el establecimiento de normar disminuirán los problemas de indisciplina y comportamiento inapropiado, alcanzando la autorregulación y la interacción social positiva con el descenso de la indisciplina, el retraimiento y el escaso entendimiento.

Los niños y niñas no cooperan en los grupos de trabajo potenciando el individualismo, perjudicando en el manejo de conductas, en la adaptación del entorno Medina \& otros (2015) considera que resulta fundamental el conocimiento del docente en el desarrollo de capacidades que prioricen los controles de conducta, crecimiento y desarrollo, también en áreas como sensorial, lenguaje y socioemocional; de forma contraria, Kimonis, Bagner, Linares, Blake, \& Rodríguez (2014) mencionan que los infantes con problemas severos de conducta son más reactivos al reforzamiento positivo que a las prácticas basadas en el castigo como norma disciplinaria, al mismo tiempo indica que es necesaria la aplicación de actividades que favorezcan en el aprendizaje social, de igual manera Martínez \& García (2018) analiza que las metodologías utilizadas en el aula promueven el respeto, fomenta la crítica, la colaboración y búsqueda de respuestas activa; Puyuelo (2001) incluye que el escaso control del freno inhibitorio perjudica en las emociones, la comunicación, el lenguaje; de esta manera que los problemas comportamentales influye de manera negativa en la autorregulación de pensamientos; Del Pozo 
(1953) la motivación al ser una actividad de interacción favorece en lo cognitivo, afectivo y social.

En el campo educativo se proyecta en el desarrollo de la inteligencia emocional, el desarrollo social, siendo el enfoque metodológico que conlleva a un cambio de paradigma en el área pedagógica y didáctica que beneficia en el freno inhibitorio, el trabajo grupal, la cooperación y socialización con el fin de fomentar la autorregulación en el proceso de enseñanza y aprendizaje; por el contrario, la escasa adaptación de niños y niñas al ambiente formativo aumenta las dificultades en la socialización, el control de emociones repercutiendo en los niveles sociales y culturales. Los docentes al no efectuar actividades que estimulen el desarrollo de la habilidad comunicativa, generan barreras en el dialogo, expresión verbal e investigación, obteniéndose que los de niños y niñas no intercambian criterios, ni mantienen buenas relaciones con las personas del entorno; frente a esta deducción Wall, Frick, Fanti, Kimonis, \& Lordos (2016) en el caso de infantes con problemas de conducta, considera que la oposición infantil se ha relacionado con la agresión, Panadero \& Alonso (2014) dicen que el escaso control de impulsos y baja autoestima, deterioran la sensación de autoeficacia y comprensión de ideas; mientras Ruiz, Gómez, \& Badía (2018) precisan que en la actualidad la inclusión beneficia en el control de emociones a través de la aceptación, el respeto y confianza.

Los niños y niñas no demuestran solidaridad con sus compañeros que presentan dificultades, siendo escaso el establecimiento de vínculos de confianza, respeto y empatía, lo que afecta en la comunicación, el desarrollo del lenguaje, las capacidades; determinándose que los problemas de comportamiento tienen origen en el insuficiente control de emociones; Araujo (2016) incluye que el factor emocional sigue siendo desvalorizado, por considerarse opuesto a lo racional; y es que, durante mucho tiempo se consideró que el coeficiente intelectual era la garantía del éxito académico de los estudiantes, mas hoy en día se ha demostrado la fuerza que tienen las emociones para impulsar o retraer las acciones hacia el aprendizaje; en tal sentido Rodríguez (2016) afirma que los procesos emocionales deben ser considerados en el campo educativo, puesto que estos no solamente ocurren en el interior del sujeto sino que también son parte de la interacción social, los cuales conforman la base central de la educación; es así como es necesario estimular los movimientos corporales y expresiones para mejorar la comunicación y transmitir información acerca del estado emocional del organismo; como principio formativo influye en la 
motivación, el estado mental y psicológico, de acuerdo con los criterios expuesto se deduce que el freno inhibitorio es un componente significativo para el comportamiento, la experiencia, el acto escolar; lamentablemente, la escasa e insuficiente realización de actividades de integración es una barrera que afecta en la enseñanza de calidad; en este sentido, el maestro debe introducir normas morales para mantener la disciplina y fortalecer la afectividad.

Los estudiantes que no intercambian juguetes $\mathrm{u}$ otros objetos, presentan dificultades en la expresión e imaginación, pasan por la etapa de egoístas, presentando conductas dominantes para cumplir con sus gustos y caprichos, es individualista y se preocupa únicamente por sus intereses; según Ceballos (2017) la salud mental, que enlaza aspectos cognitivos y emocionales, la cual busca la excelencia personal, el ajuste psicosocial y el cumplimiento de tareas evolutivas, por el contrario (Morales \& Rodríguez, 2017) establece la necesidad de generar en actividades lúdicas y pedagógicas que divierta a los infantes y genere aprendizajes significativos desde una pedagogía que se fundamente en la diversidad desde una ética dialógica; desprendiéndose que el entorno educativo ofrece oportunidades para aprender a colaborar, compartir sus juguetes, respetar su turno, obedecer, cumplir reglas, participar con entusiasmo, es así como, la familia y la escuela estimulan en el niño o niña el desarrollo de sus capacidades de autorregulación emocional, de diferenciación entre sentir, pensar y hacer, que mediante la comprensión de reglas afianza sus conocimientos, la adaptación e integración. Gallardo \& Ruíz (2018), los niños y niñas, al ser conscientes de sus propios estados internos, serán capaces de comprender los sentimientos de quienes los rodean, tolerar presiones ambientales y frustraciones, desarrollar habilidades sociales y de trabajo en equipo

\section{Conclusión}

De los resultados obtenidos se determina que es necesaria la sistematización de una base teórica para respaldar la importancia del freno inhibitorio en la autorregulación, considerando que es insuficiente la estimulación del freno inhibitorio y autorregulación, siendo así que los niños y niñas presentan dificultades en el control de los movimientos coordinados de brazos, piernas y torso, lo que afecta en el desarrollo cognoscitivo, el lenguaje, atención, el comportamiento y rendimiento académico. En el análisis del nivel del freno inhibitorio en la autorregulación en el sistema educativo se establece que los niños y niñas son intranquilos, determinando que la 
escuela no responde a las necesidades educativas de aprendizaje y crecimiento, no estimula ni activa la atención selectiva de los niños y niñas, como resultado se desplazan y hablan en forma continua generando conflictos entre ellos; la mayoría no reconoce su lateralidad, la organización de diferencias espaciales /derecha izquierda, lo que desfavorece en la estimulación del dominio del esquema corporal y la orientación espacial. Además, los infantes reaccionan de forma negativa ante un impulso de sus compañeros, no dominan las emociones y conductas, es escaso el autocontrol y confianza en sí mismo, generando limitaciones en el desarrollo social, emocional y cognoscitivo.

\section{Referencias Bibliográficas}

Aldrete, C., Carrillo, P., Mansilla, A., Schnaas, 1., \& Esquivel, F. (2013). De la regulación emocional y cognitiva a la autorregulación durante el primer año de vida. (file://C:/Users/PC146/Downloads/10630-18136-1-PB.pdf, Ed.) Anuario de Psicología/The UB Journal of Psychology.

Araque, N. (8 de diciembre de 2017). En la educación emocional en el proceso educativo inicial en Ecuador $\mathrm{y}$ España. (http://investigacion.utc.edu.ec/revistasutc/index.php/utciencia/article/view/35/36, Ed.) UTCIENCIA, Ciencia y Tecnología al servicio del pueblo. Latindex, 2(3), 12.

Araujo, M. (Marzo de 2016). En la emoción como principio pedagógico en el proceso de enseñanza y aprendizaje. (file:///C:/Users/PC-146/Downloads/10056-30094-1-SM.pdf, Ed.) Revista Ontosemiótica. Laboratorio de Investigaciones Semióticas y Literarias (LISYL), 7.

Avanzini, G. (2016). En Problemas de Pedagogía. El Fracaso escolar. (https://www.amazon.com/El-Fracaso-Escolar-Guy-Avanzini/dp/B072K9FWMN, Ed., \& L. Medrano, Trad.) Barakaldo, España: Herder, S.A.

Cano, R., \& Casado, M. (18 de Agosto de 2015). En la Escuela y familia. Dos pilares fundamentales para unas buenas prácticas de orientación educativa a través de las escuelas de padres. (http://www.redalyc.org/pdf/2170/217036214003.pdf, Ed.) Revista Electrónica Interuniversitaria de Formación del Profesorado, 18(2), 15. 
Castro, B., \& Casado, O. (Enero de 2014). En la autorregulación en Educación Infantil. (http://revistas.uv.cl/index.php/IEYA/article/viewFile/759/735, Ed.) Index, 3(2), 5.

Ceballos, Z. (2017). En el Bienestar psicológico en los niños, retos de la familia y la institución educativa. (file://C:/Users/PC-146/Downloads/697-1873-1-SM.pdf, Ed.) Tarbiya, Revista de Investigación e Innovación Educativa, 11.

Cladellas, R., Clariana, M., \& Badía, M. (Diciembre de 2015). En la Indisciplina Instruccional y Convencional: $\mathrm{Su}$ Predicción en el Rendimiento Académico. (http://www.scielo.org.co/pdf/rcps/v24n2/v24n2a06.pdf, Ed.) Scielo, 2(2), 14.

Del Pozo, A. (1953). Generosidad y egoísmo. Revista Española de Pedagogía, 11(42), 12.

Fonagy, P., Gergely, G., Jurist, E., \& Target, M. (2002). En la regulación afectiva, la mentalización $\mathrm{y}$ el desarrollo del self. (http://www.aperturas.org/articulos.php?id=0000558\&a=La-regulacion-afectiva-lamentalizaciony-el-desarrollo-del-self, Ed.) Psychoanalytic Inquiry (30), 22.

Gallardo, M., \& Ruíz, J. (Febrero de 2018). En Las estrategias socio afectivas desde la perspectiva pedagógica y didáctica para mejorar las emociones de estudiantes de cinco años. (https://www.magisterio.com.co/articulo/estrategias-didacticas-y-actividades-para-la-educacionemocional-en-el-aula, Ed.) Ciencia y Tecnología, 12.

García, L., Naissir, L., Contreras, C., \& Moreno, A. (Julio de 2015). En el estado emocional y el bajo rendimiento académico en niños $y$ niñas de Colombia. (http://www.unife.edu.pe/publicaciones/revistas/psicologia/2015_1/Leslie_Bravo.pdf, Ed.) Indizada Latindex. Avances en psicología. Revista de la Facultad de Psicología y Humanidades Volumen y en la Base de datos EBSCO, 23(1), 8.

García, M., \& Barrio, G. (24 de Abril de 2015). En el Afrontamiento de situaciones conflictivas en un grupo-clase de Educación Infantil. Una investigación cualitativa etnográfica. (file://C:/Users/PC-146/Downloads/4868-24192-1-PB.pdf, Ed.) Oredalei, 4(1), 26.

Jaqueira, A., Lavega, P., Lagardera, F., Araujo, P., \& Rodrigues, M. (1 de Marzo de 2014). Educando para la paz jugando: Género y emociones en la práctica de juegos cooperativos 
competitivos.

(file://C:/Users/PC-146/Downloads/194071-699101-2-PB\%20(1).pdf,

Ed.)

Educación Siglo XXI, 32(1), 18.

Jaume, M., Ribot, M., \& Mas, C. (27 de Julio de 2018). En la organización del espacio por ambientes de aprendizaje en la Educación Infantil: significados, antecedentes y reflexiones. (http://www.usc.es/revistas/index.php/reladei/article/view/4726/5081,

Ed.)

Revista Latinoamericana de Educación Infantil. Reladei, 3(2), 21.

Jiménez, A. (2 de Febrero de 2018). En la Inteligencia emocional. (https://www.aepap.org/sites/default/files/457-469_inteligencia_emocional.pdf, Ed.) Lúa, 13.

Kimonis, Bagner, Linares, Blake, \& Rodríguez. (19 de Abril de 2014). En Rasgos sin emociones y el tratamiento de los problemas de conducta en la infancia y la adolescencia: una revisión exhaustiva. (https://link.springer.com/article/10.1007\%2Fs10567-014-0167-1, Ed.) Revisión clínica de psicología infantil y familiar, 7(3), 20.

Kopp, C. (. (Marzo de 1982). Antecedentes de autorregulación: una perspectiva de desarrollo. Developmental Psychology, 18(2), 14.

Martínez, A., \& García, M. (31 de Enero de 2018). En el Aprendizaje basado en proyectos en educación infantil: cambio pedagógico y socia. Revista Iberoamericana de Educación, 20.

Medina, M., Kahn, I., Muñoz, P., Leyva, J., Moreno, J., \& Vega, S. (Julio de 2015). En el Neurodesarrollo infantil: características normales y signos de alarma en el niño menor de cinco años. (https://www.scielosp.org/pdf/rpmesp/2015.v32n3/565-573/es, Ed.) Scielo. Revista Peruana de Medicina Experimental y Salud Publica, 32(3), 9.

Morales, S., Arriaga, D., Peña, M., \& Mandujano, J. (18 de Octubre de 2017). En Prácticas de crianza predictoras de problemas moderados de conducta en niños. Revista internacional de investigación en adicciones, 3(2), 14.

Morales, S., Félix, V., \& Rosas, M. (Mayo de 2015). En el Incremento en la resistencia al cambio por reforzamiento de conducta alternativa: un estudio traslacional. Revista Mexicana de Análisis de la Conducta. (http://www.scielo.org.co/pdf/apl/v33n1/v33n1a05.pdf, Ed.) Scielo. Revista mexicana de análisis de la conducta, 40(1), 20. 
Morales, S., Romero, V., Rosas, M., López, F., \& Nieto, J. (2015). En las Prácticas de crianza asociadas al comportamiento negativista desafiante $y$ de agresión infantil. (http://www.redalyc.org/html/799/79933768005/, Ed.) Avances en psicología latinoamericana, 33(1), 19.

Muñoz, L. (2017). En La autorregulación y su relación con el apego en la niñez. (http://www.scielo.org.co/pdf/rlcs/v15n2/v15n2a03.pdf, Ed.) Revista Latinoamericana de Ciencias Sociales, Niñez y Juventud, 15(2), 15.

Navea, A. (29 de Diciembre de 2017). En el aprendizaje autorregulado en estudiantes de ciencias de la salud: recomendaciones de mejora de la práctica educativa. (file:///C:/Users/PC146/Desktop/AA\%20AUTORREGULACI\%C3\%93N.pdf, Ed.) ELSEVIER. Educación Médica, 8.

Panadero, E., \& Alonso, J. (20 de Mayo de 2014). Teorías de autorregulación educativa: una $\begin{array}{llll}\text { comparación } & \text { y } & \text { reflexión teórica. } & \text { (file:///C:/Users/PC- }\end{array}$ 146/Downloads/S1135755X14000037_S300_es.pdf, Ed.) Psicología Educativa, 12.

Páramo, M., Martínez, M., \& Raposo, M. (Noviembre de 2015). Instrumentos de una investigación en desarrollo sobre el aprender a aprender: descripción y posibilidades. (http://revistas.udc.es/index.php/reipe/article/view/184, Ed.) Revista de Estudios e Investigación en Psicología y Educación, 06, 6.

Puyuelo, M. (2001). Psicología, audición y lenguaje en diferentes cuadros infantiles. Aspectos comunicativos y neuropsicológicos. Revista Neurológica. Elsevier, 32(10), 6.

Regni, R. (18 de Diciembre de 2014). La polarización de la atención y las armas de distracción masiva. (http://www.usc.es/revistas/index.php/reladei/article/view/4747, Ed.) RELAdEI. Revista Latinoamericana de Educación Infantil, 3(3), 12.

Ríos, J. A., Álvarez, L., Sierra, D., \& Zuleta, A. (17 de Enero de 2017). Influencia del nacimiento pretermino en procesos conductuales y emocionales de niños en etapa escolar primaria. (http://revistaumanizales.cinde.org.co/rlcsnj/index.php/Revista-

Latinoamericana/article/view/2681, Ed.) Revista Latinoamericana de Ciencias Sociales. Revista Manizales, 1. 
Rodríguez. (28 de Junio de 2016). Las emociones en el proceso de enseñanza-aprendizaje. (file://C:/Users/PC-146/Downloads/emociones-proceso-ensenanza-aprendizaje.pdf, Ed.) Revista Vinculando Desarrollo Sostenible + Desarrollo Personal, 13.

Ruiz, B., Gómez, M. F., \& Badía, M. (22 de Junio de 2018). Influencia de la calidad de vida, la participación y la resiliencia en el desarrollo de alumnos de educación primaria. (file://C:/Users/PC-146/Downloads/Dialnet-

InfluenciaDeLaCalidadDeVidaLaParticipacionYLaResil-6231796.pdf, Ed.) Revista Española de Discapacidad, 5(2), 18.

Sáiz, M., \& Pérez, M. (29 de Abril de 2016). Autorregulación y mejora del autoconocimiento en resolución de problemas. (http://www.redalyc.org/articulo.oa?id=21345152002, Ed.) Psicología desde El Caribe, 1, 15.

Taboada, R., \& Pinto, B. (Marzo de 2018). Evaluación neuropsicológica en dos niños con trastorno por déficit de atención e hiperactividad: estudios de caso. (http://www.scielo.org.bo/pdf/rap/v16n1/v16n1_a04.pdf, Ed.) Scielo, 16(1), 41.

Torrano, F., Fuentes, J., \& Soria, M. (Junio de 2017). Aprendizaje autorregulado: estado de la cuestión $\mathrm{y} \quad$ retos psicopedagógicos. (http://www.scielo.org.mx/scielo.php?script=sci_arttext\&pid=S0185-26982017000200160, Ed.) Scielo. Perfiles educativos, 39(156), 14.

Tourón, J., Santiago, R., \& Díez, A. (Marzo de 2015). Cómo convertir la escuela en un espacio de aprendizaje. (http://www.digital-text.com/wp-content/uploads/2015/03/FlippedClassroom.pdf, Ed.) The Flipped Classroom. Grupo Océano.

Urbieta, P., \& Esteinou, I. (1 de Febrero de 2017). Compendio de lecturas educando sin violencia. Técnicas para manejo del estrés e inteligencia emocional. (http://www.ririki.org.mx/publicaciones/21.Manual-Educando_sin_violencia.pdf, Ed.) Ririki Intervención social. SEDESO., 33.

Villanueva, S. (24 de Octubre de 2016). Alfa Cronbach, Prueba de regresión lineal, Prueba T, Chi cuadrada \& Coeficiente de correlación de Pearson. Pedagogía, 12. 
Wall, Frick, Fanti, Kimonis, \& Lordos. (16 de Diciembre de 2016). Examen de la psicopatía exitosa. (https://pdfs.semanticscholar.org/672f/10b89baca717ef26f97bd78eaa412b6df409.pdf, Ed.) Scholar Word @1, 51. 